\title{
The Use of Computational Fluid Dynamics to Assess the Hull Resistance of Concept Autonomous Underwater Vehicles
}

\author{
Alexander Phillips ${ }^{1}$, Maaten Furlong ${ }^{2}$, Stephen R. Turnock ${ }^{1}$, member, IMechE \\ ${ }^{1}$ University of Southampton, ${ }^{2}$ National Oceanography Centre, Southampton \\ abp@soton.ac.uk, maatenf@noc.soton.ac.uk, s.r.turnock@soton.ac.uk
}

\begin{abstract}
Autonomous Underwater Vehicles (AUV's) provide an important tool for collecting detailed scientific information from the oceans depths. The hull resistance of an AUV is an important factor in determining the powering requirements and range of the vehicle. This paper discusses the use of Computational Fluid Dynamics (CFD) to determine the hull resistance of three existing AUV's, of differing shape and size. The predictions are compared with available experimental data and good agreement found. This work has demonstrated that with use of suitable shape parameterisation it is possible to carry out concept design evaluation using a RANS flow solver.
\end{abstract}

\section{INTRODUCTION}

The applications of Autonomous Underwater Vehicles (AUVs) are diverse, [1] ranging from :-

- scientific research (e.g. ocean sampling and environmental monitoring);

- commercial uses including pipeline inspection and cable surveys;

- military applications such as mine hunting.

Since the AUV must carry its power source, a good understanding of both the propulsion and hotel loads is required at the early design stage. Evaluating the hydrodynamic drag of a prototype AUV hull form is expensive and time consuming if carried out using either experimental facilities (towing tanks, circulating water channels, wind tunnels) or computational fluid dynamics (CFD), which requires an experienced and skilled user for reliable results. The eventual aim of the program of work under way is to develop specific AUV hull concept design techniques that are robust and reliable. To this end, CFD analysis methods are being investigated which combine automated meshing and parametric hull shape definitions to reduce overheads when evaluating the design of a concept AUV hull.

As part of the design process computational studies of the fluid flow around three AUV's (see Figure 1) have been performed to determine the hydrodynamic drag experienced by existing vehicles for validation against existing model test and full scale experimental data. The objectives of this study are: (1) to demonstrate the application of CFD to determine the hull resistance of AUV's; (2) benchmark the computational results against existing experimental results; (3) demonstrate the application of geometry parametrisation suitable for design optimisation.

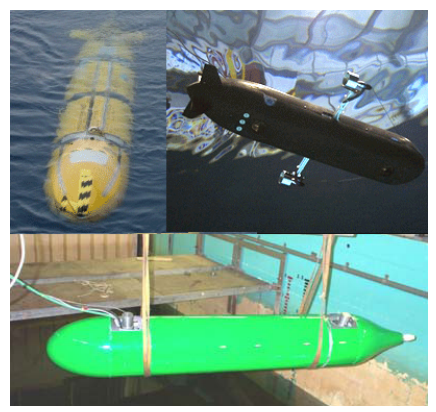

Fig. 1. Autosub (top left), Soton-AUV (top right) \& C-Scout (bottom)

\section{EMPIRICAL DRAG ESTIMATES FOR AUV DESIGN}

The drag experienced by an axi-symmetric AUV operating away from the surface and travelling in a straight line is a direct result of the viscosity of the water. The viscous effects are traditionally split into two components: the skin friction drag due to the viscous shear of the fluid flowing along the hull; the form drag due to the development of the boundary layer and the resulting differential pressure distribution fore and aft along the hull.

Empirical relationships are available which allow initial estimates of the drag. The ITTC 57 correlation line is commonly used in surface ship design to estimate the skin friction $\left(C_{F}\right)$ component of the viscous drag as a function of Reynolds number $\left(R_{N}\right)$ :

$$
C_{F_{1957}}=\frac{0.075}{\left(\log \left(R_{N}\right)-2\right)^{2}}
$$

This is multiplied by a form factor $(1+k)$ to provide an estimate of the complete viscous drag coefficient. The magnitude of the form factor is a function of the hull shape. Hoerner [2] proposed the following equation to estimate the form factor for a streamlined body as a function of vessel length (l) and diameter (d).

$$
(1+k)=1+1.5(d / l)^{3 / 2}+7(d / l)^{3}
$$

This simple approach provides an initial estimate of the powering requirements, although the value of the form factor is hull shape dependent. For new or novel hull forms, determining the value of $(1+\mathrm{k})$ using empirical methods adds uncertainty to the drag estimate. 


\section{CFD Methodology}

The fluid flow around the three existing AUVs has been modelled using the commercial finite volume code ANSYS CFX 10 (CFX) [3]. For these calculations the fluid's motion is modelled using the incompressible (3), isothermal Reynolds Averaged Navier Stokes (RANS) equations (4) in order to determine the cartesian flow field $\left(u_{i}=u, v, w\right)$ and pressure (p) of the water around an AUV hull:

$$
\begin{gathered}
\frac{\partial \overline{U_{i}}}{\partial x_{1}}=0 \\
\frac{\partial \overline{U_{i}}}{\partial t}+\frac{\partial \overline{U_{i} U_{j}}}{\partial x_{j}}=-\frac{1}{\rho} \frac{\partial P}{\partial x_{i}}+\frac{\partial}{\partial x_{j}}\left\{\nu\left(\frac{\partial \overline{U_{i}}}{\partial x_{j}}+\frac{\partial \overline{U_{j}}}{\partial x_{i}}\right)\right\}-\frac{\partial \overline{u_{i}^{\prime} u_{j}^{\prime}}}{\partial x_{j}}+f_{i}
\end{gathered}
$$

\section{A. Parametrisation}

The rapid production of high quality grids for a parametric series of AUV hulls is desirable if a quality optimisation process is to be performed. For this study, the meshes are produced by careful parameterisation of the AUV hull (see Figure 2) using script files for driving the meshing package ANSYS ICEM CFD. These produce high quality multi-block structured grids with detailed control over the essential mesh parameters.

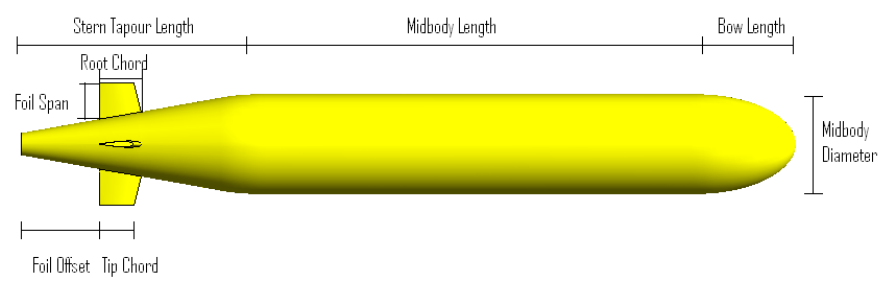

Fig. 2. Parameterised Autosub hull form.

\section{B. Physics Definition and Mesh Independence}

In order to ensure sufficient grid quality parametric studies were performed to investigate the sensitivity of the solution to: number of finite volume hexahedra; distance between the body and the domain boundaries; mesh size in wall boundary layer and solver convergence criteria.

1) Domain Boundaries: Solution of the Navier Stokes equations is only possible over a finite domain. In practice this requires placing inlet and outlet and wall boundary conditions to define the domain. Correct positioning of the boundaries is vital to ensure the flow is not artificially constrained and is equivalent to blockage effects in tank testing.

Constraining the flow increases the predicted resistance, Figure 3, while maintaining the same mesh density results in the less constrained meshes having a much higher number of elements; a suitable compromise is required, and for example with Autosub simulations a wall distance of $4 \mathrm{~m}$ was selected.

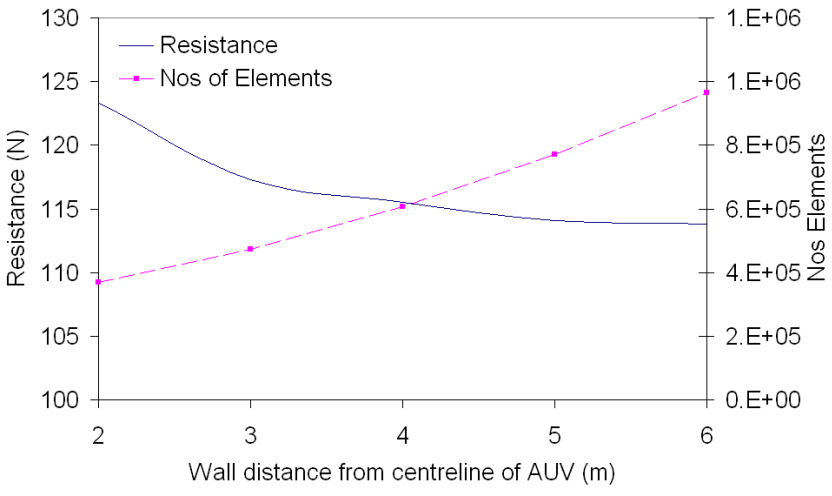

Fig. 3. Influence of boundary locations on resistance estimates.

TABLE I

Autosub DRAG AT $2 \mathrm{~m} / \mathrm{s}$

\begin{tabular}{|c|c|c|c|}
\hline Mesh Density & No(s) Elements & Run Time (min) & Drag (N) \\
\hline Coarse & 163,448 & 34 & 117.7 \\
\hline Medium & 606,526 & 129 & 115.5 \\
\hline Fine & $1,264,756$ & 154 & 114.9 \\
\hline
\end{tabular}

2) Mesh Density: An appropriate mesh density is vital to achieve a cost effective solution since solution accuracy and run time are both heavily dependent on the number of cells. Table I gives an example grid convergence study.

The area of interest for this investigation is the resistance which is generated due to viscous effects in the boundary layer. To reduce the number of elements required to define the boundary layer wall functions where used to model the fluid behaviour in the boundary layer region. As a general guideline, a boundary layer should be resolved with at least 10 nodes within the boundary layer when using wall functions, with a first layer thickness of between $20 \leq \Delta y^{+} \leq 200$ [3]. These guidelines have been adhered to when producing meshes.

3) Turbulence Models: By time averaging the Navier Stokes equations to generate the RANS equations, 6 further unknowns have been created, termed the Reynolds stresses: $\frac{\partial \overline{u_{u}^{\prime} u_{j}^{\prime}}}{\partial x_{j}}$. Various turbulence models have been proposed to provide solutions to the Reynolds stresses in terms of known quantities to allow closure of the RANS equations [4]. Different turbulence models have been tailored to different types of turbulent flows. The $k-\epsilon$ model is a commonly used turbulence model for engineering simulations due to its robustness and application to a wide range of flows. However it is known to be poor at locating the onset and extent of separation [5]. An Alternative the Shear Stress Transport (SST) model is better at predicting separation [5] likely to be found at the aft of the AUV.

For the bare Autosub hull the drag predictions from the SST and $k-\epsilon$ turbulence model give a very high level of correlation, and the $k-\epsilon$ model is used for the remainder of results presented. 
4) Simulation Numerical Uncertainty: A full set of mesh sensitivity studies have been performed to establish a simulation numerical uncertainty $\left(U_{S N}\right)[6]$ associated with the definition of the fluid domain, selection of turbulence model and convergence criteria. For the Autosub simulations, $U_{S N}$ was found to be $\pm 3 \%$.

\section{Computer Simulation}

Simulations were run on a high specification desktop pc running 64 bit Windows XP with 4 GB of RAM. Solutions presented have been calculated using the high resolution advection scheme. The residual mass error was reduced by four orders of magnitude and lift and drag forces on the AUV were monitored to ensure convergence. Typical run times were wall clock two hours for completely submerged cases, and twelve hours for simulations including the free surface.

\section{EXPERIMENTAL DATA}

Confidence in the computational results can be achieved by benchmarking the solutions against existing experimental or analytical results. Experimental testing of AUV's in towing tanks modifies the fluid flow compared to open ocean conditions in two respects:

- Towing tanks have limited dimensions, effectively creating a constrained channel, modifying the fluid flow around the AUV compared to open water conditions. This may lead to wave resistance if the model is too close to the free surface, or blockage effects if the walls of the tank significantly restrict flow round the hull.

- Fixing the model in position by support posts; the drag of the posts and any drag induced by interactions of the post and model are recorded by the dynamometer and must be stripped from the measured drag.

These issues, along with experimental error, must be accounted for when using experimental results. CFD can be used to replicate both constrained experimental conditions and operating open ocean conditions, allowing validation of the CFD and improved understanding of the influence of experimental conditions on resistance predictions.

\section{Autosub}

The Autosub family of AUVs has been exploring the oceans since 1996. They have been developed by a team of engineers and oceanographers at the National Oceanography Centre, Southampton. Autosub has been employed in scientific research projects ranging from mapping manganese distributions in a sea loch to ground breaking under ice exploration in the Arctic and Antarctic [7] [8]. Autosub's principle dimensions are listed below:

- Length $7 \mathrm{~m}$

- Diameter $0.9 \mathrm{~m}$

- Speed Range $1.0-2 \mathrm{~m} / \mathrm{s}$

- Operating $R_{N} 5.9 \times 10^{6}-11.8 \times 10^{6}$

Model scale tests were performed on a $2 / 3$ rd scale model of the Autosub hull form by Kimber et al. [9] at the HASLAR facility $(270 \mathrm{~m} \times 12.2 \mathrm{~m} \times 5.5 \mathrm{~m}$ deep $)$. Further tests were performed by Fallows [10] on a $2.5 \mathrm{~m}$ scale model of Autosub at the Solent University Towing Tank $(60 \mathrm{~m} \times 3.7 \mathrm{~m} \times 1.8$ $\mathrm{m}$ deep). Figure 4 compares experimental, empirical and CFD results for skin friction and total resistance.

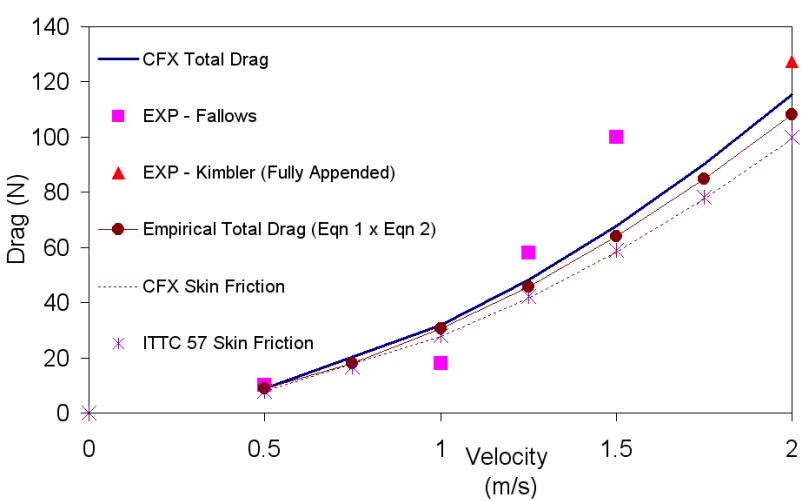

Fig. 4. Comparisons of drag predictions for Autosub

There is significant scatter in the experimental results although the trend agrees with the CFD predictions. Fallow's experiments were performed with the model 2.6 diameters from the free surface and did experience wave making drag. The skin friction predictions from CFD and the ITTC 57 line give high correlation. The form factor predicted by equation 2 of 1.084 for a streamlined body is lower than the 1.157 from the CFD result. The empirical result does not include the effects of separation at the transom stern.

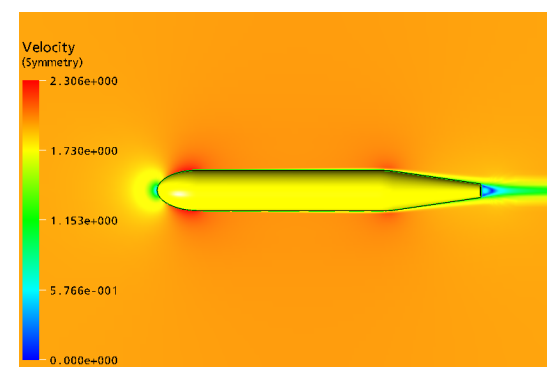

Fig. 5. Magnitude of velocity field around Autosub hull at $2 \mathrm{~m} / \mathrm{s}$

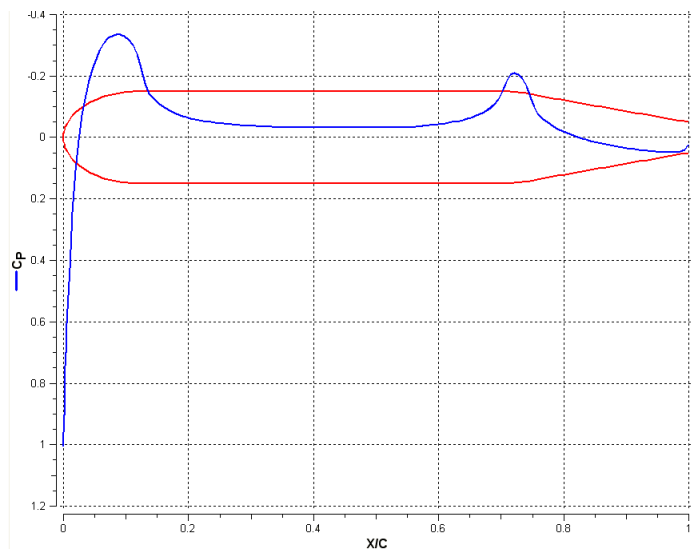

Fig. 6. Pressure distribution around the naked Autosub hull at $2 \mathrm{~m} / \mathrm{s}$ 
As shown in Figs 5 and 6, detailed information about the velocity and pressure distribution $C_{P}=\frac{P-P_{0}}{1 / 2 \rho U_{0}^{2}}$ around the hull can be extracted from CFD simulations; from a stagnation point at the bow, the flow is accelerated around the shoulder of the hull up to a maximum velocity of $1.3 U_{0}$. Along the parallel mid-body the boundary layer grows, the flow is accelerated as it reaches the stern taper, where the boundary layer grows rapidly becoming thicker. Large vortical structures form behind the stern which form the wake region.

AUV's are manoeuvered using thrusters, control surfaces or a combination of both. The addition of control surfaces modifies the fluid flow around the AUV and so modify the drag. Autosub is controlled by four movable control surfaces mounted at the rear of the vessel in a cruciform arrangement. Two vertical rudders control the yaw of the vessel, while two horizontal stern planes adjust the pitch of the vessel. Kimber et al [9] investigated the influence of three different sets of control surfaces on the drag and manoeuvrability of Autosub.

Table II compares the appended hull resistance from model tests with CFX results for $2 \mathrm{~m} / \mathrm{s}$ full scale speed. The CFD predictions closely follow the experimental results, the small discrepancies can be attributed to the CFD model ignoring the influence of the 'auto-rotating' propeller present in the experiments. This demonstrates CFD's ability to assess the change in drag due to small variations in geometry.

TABLE II

Appended Autosub Drag Predictions at $2 \mathrm{~m} / \mathrm{s}$ [9]

\begin{tabular}{|c|c|c|}
\hline $\begin{array}{c}\text { Appendage } \\
\text { set }\end{array}$ & $\begin{array}{c}\text { Experimental } \\
\text { Full Scale Drag (N) }\end{array}$ & $\begin{array}{c}\text { CFD } \\
\text { Full Scale Drag (N) }\end{array}$ \\
\hline Bare Hull & - & 115 \\
\hline A & 128 & 124 \\
\hline B & 133 & 127 \\
\hline C & 142 & 132 \\
\hline
\end{tabular}

One of the key advantages of using CFD is the ability to parameterise the hull shape and consequently rapidly assess the hull resistance of a series of AUV's at the initial design phase. Autosub has a 2:1 elliptical bow. Using the parametric model of Autosub, the influence of bow shape can rapidly be determined using CFD. Three bow shapes have been considered for a constant hull length: a circular bow, a 2:1 ellipsoid bow and a 3:1 ellipsoid bow. The more streamlined hull will have the lower drag but it also has a reduced parallel mid section convenient for placing the pressure vessel. Figure 7 demonstrates the reduction in hull resistance associated with a more streamlined bow. Figure 8 illustrates the influence of bow shape on the pressure distribution.

Modifying the shape of the bow modifies the skin friction and pressure drag experienced by the vehicle. Variation in the skin friction drag can be predicted with use of Eqn (4). Many empirical methodologies for determining $(1+\mathrm{k})$ only consider the length and diameter. By making the bow more streamlined, the pressure drag is reduced, see Table III.

This form of study at the early design stage provides useful information to the designer when trying to balance the conflicting requirements of a streamlined hull for good

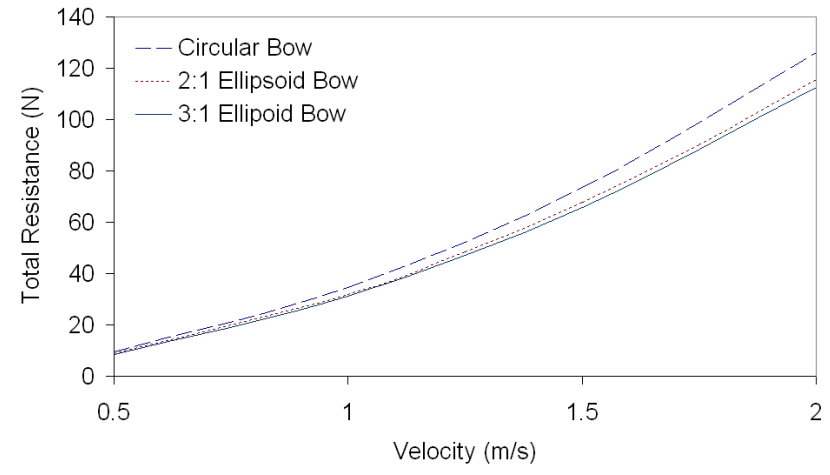

Fig. 7. Comparison of total drag For parameterised Autosub

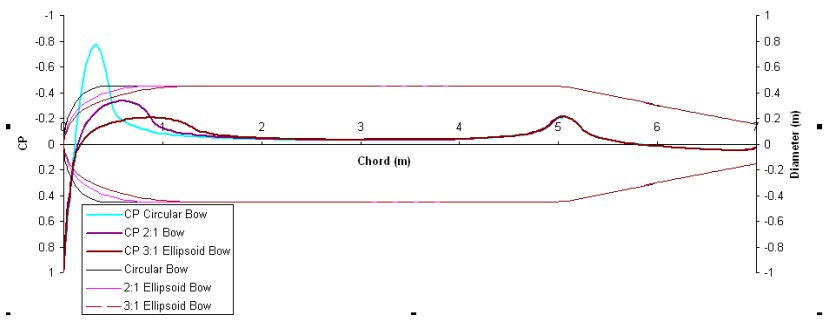

Fig. 8. Comparison of pressure distribution For parameterised Autosub

hydrodynamic performance against a fuller hull for increased payload [11].

The goal when selecting a hull form, propulsion system and power source is optimum endurance of the AUV while performing its required mission. This may not result in the lowest drag hull form but the best integrated hull shape and propulsion device. CFD, while eliminating some of the uncertainty inherent in empirical approaches, also provides detailed information about the fluid flow around the concept hull, allowing the propeller to be correctly matched to the hull form.

The average speed of the flow into the propeller $\left(V_{a}\right)$ is related to the vessel speed $\left(V_{s}\right)$ by the Wake Fraction $w_{T}$ :

$$
\text { Taylor Wake Fraction }=w_{T}=\frac{\left(V_{s}-V_{a}\right)}{V_{s}}
$$

A knowledge of the average $w_{T}$ at any given radius is required for detailed propeller design such as radial pitch variation. Fluctuations in the $w_{T}$ as the propeller rotates lead to cyclic load variations which has implications for propeller strength and vibrations.

The average Wake Fraction over the propeller disk can be deduced indirectly from standard open water and self propulsion tests. For more detailed information, detailed wake surveys can be performed using expensive experimental techniques to measure the wake directly (e.g. pitot tubes or Laser Doppler Anemometry).

The mean Wake Fraction over the propeller disk can easily be extracted from a CFD analysis. For the naked Autosub $w_{T}=0.17$ and for the appended Autosub $w_{T}=0.19$. Figure 9 illustrates the fluid velocity at the location of the propeller. 
TABLE III

FORM FACTOR VARIATION

\begin{tabular}{|c|c|}
\hline Bow Shape & Form Factor \\
\hline Circular & 1.256 \\
\hline 2:1 Ellipsoid & 1.157 \\
\hline $3: 1$ Ellipsoid & 1.136 \\
\hline
\end{tabular}

The thickness of the boundary layer is clearly visible along with the wake from the control surfaces, which is seen as a cruciform of slower moving fluid.

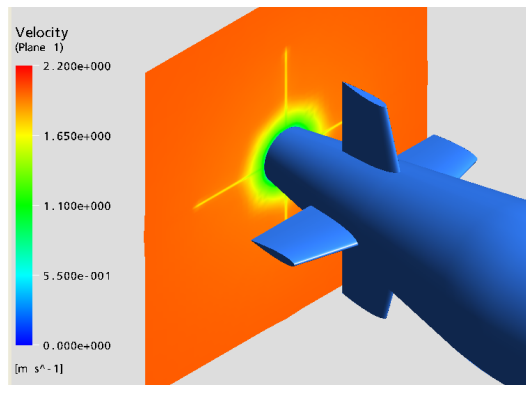

Fig. 9. Wake pattern in the propeller region of appended Autosub

\section{C-ScOUT}

The Canadian Self Contained Off-the Shelf Underwater Testbed (C-Scout) is a torpedo shaped AUV developed by a team of students and graduates at the Memorial University of Newfoundland and Labrador as a testbed for underwater research. The C-Scout project aims to develop AUV for environmental monitoring missions [12]:

- Length $2.7 \mathrm{~m}$

- Diameter $0.4 \mathrm{~m}$

- Speed Range 0.5 to $2.5 \mathrm{~m} / \mathrm{s}$

- Operating $R_{N} 1.1 \times 10^{6}-5.7 \times 10^{6}$

Bose et al. performed straight line resistance tests on the full scale C-Scout vehicle at the Memorial University Towing Tank [12]. They established that the experimental results include significant wave resistance at speeds of greater than $2 \mathrm{~m} / \mathrm{s}$. To ensure negligible wave resistance, submerged bodies should be at least five diameters below the free surface [2]. Restrictions in the test facilities resulted in C-Scout being positioned 2.25 diameters below the free surface.

To capture the wave making resistance component, it is possible to run a multiphase flow and simulate the free surface. This is more computationally intensive and special care must be taken with the size of the domain and the meshing strategy.The blocking strategy was adjusted to ensure that additional nodes were placed $\pm 0.2 m$ of the still water free surface to aid capture of the free surface.

While specifying the inlet and outlet boundary conditions, the free surface elevations are fixed at these locations. If the boundaries are too close to the body this can modify the local wave pattern, changing the wave making drag substantially. These simulations have been performed with the inlet and outlet ten bodylengths away from the AUV. With the lateral boundaries set at the locations of the tank walls.
Figure 10 shows the calculated free surface elevation for CScout at $3 \mathrm{~m} / \mathrm{s}$. A bow wave is clearly present as a region of elevated free surface above the bow. A trough is also present over the stern of the vessel.

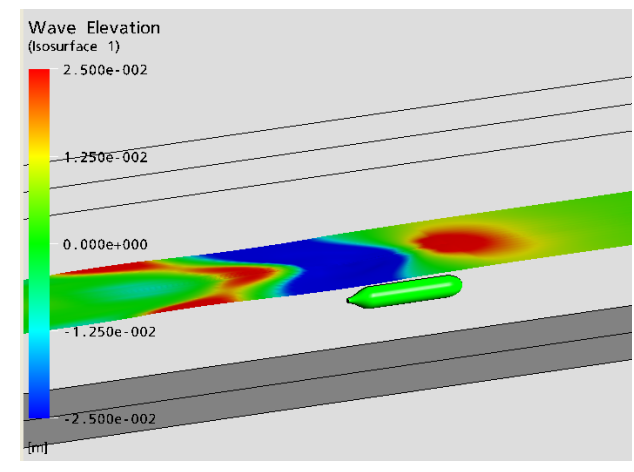

Fig. 10. C-Scout's wave pattern at $3 \mathrm{~m} / \mathrm{s}$

Figure 11 shows the drag coefficients $\left(C_{D}\right)$ for the C-Scout vehicle. For a completely submerged AUV, the viscous drag coefficients would be expected to follow a similar shape to the ITTC 57 skin friction line. The rapid rise in experimental $C_{D}$ after a $R_{N}$ of $1.5 \times 10^{6}$ is similar to the wave making drag component present for surface ships. Drag coefficients defined in this paper are determined based on the frontal projected area $\left(A=\pi d^{2} / 4\right)$ :

$$
C_{D}=\frac{\text { Drag }}{1 / 2 \rho A U^{2}}
$$

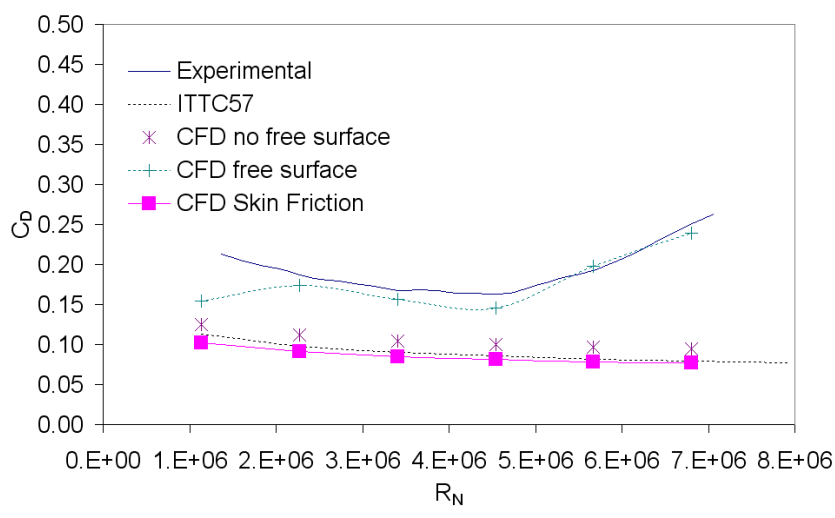

Fig. 11. Comparison of drag coefficients for C-Scout

Figure 12 compares empirical and experimental results for straight line resistance of C-Scout with CFD simulations with and without a free surface.

The CFD simulations including the free surface show a good level of correlation with the experimental values, while the fully submerged CFD case illustrates the large influence free surface effects can have on drag predictions of AUV's.

\section{SOTON AUV}

SOTON AUV was developed by a team from the University of Southampton to compete in the Student Autonomous Underwater Challenge - Europe (SAUC-E). Fully appended 


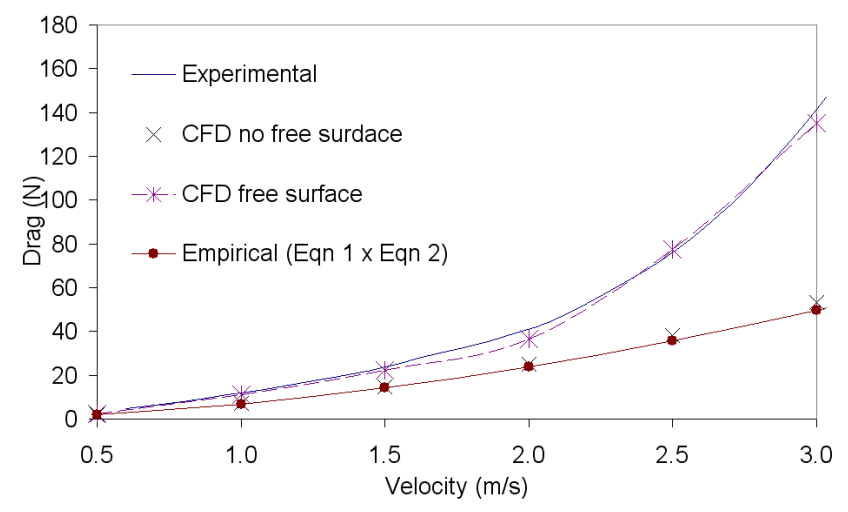

Fig. 12. Comparison of total drag for C-Scout

straight line resistance tests were performed by Andersen et al. on the SOTON AUV [13]. The principle dimensions of SOTON AUV are listed below: -

- Length $1.33 \mathrm{~m}$

- Depth $0.219 \mathrm{~m}$

- Breadth $0.269 \mathrm{~m}$

- Speed Range $0.2-1.5 \mathrm{~m} / \mathrm{s}$

- Operating $R_{N} 2.2 \times 10^{5}-1.7 \times 10^{6}$

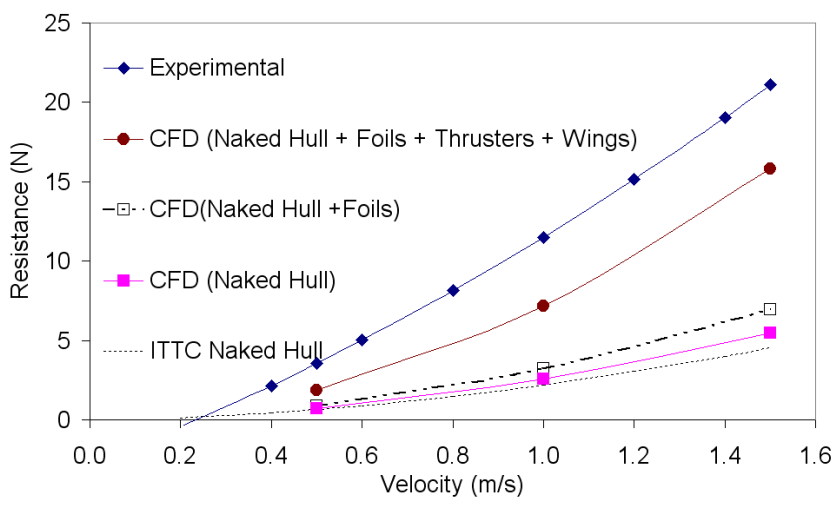

Fig. 13. Drag predications for SOTON AUV

TABLE IV

DRAG BREAKDOWN FROM CFD ANALYSIS FOR SOTON AUV AT $1.5 \mathrm{~m} / \mathrm{s}$

\begin{tabular}{|c|c|}
\hline Component & Drag (N) \\
\hline Naked Hull & 5.46 \\
Foils & 1.50 \\
Tunnel Thrusters & 0.50 \\
Wings & 7.45 \\
\hline Total & 15.83 \\
\hline
\end{tabular}

SOTON AUV was tested prior to manufacture of it's faired wings. Consequently, the appendage drag for SOTON AUV is considerable in relation to the naked hull resistance. The wings used for the model tests comprised of $2 \mathrm{~cm} \times 2 \mathrm{~cm}$ unfaired aluminium extrusion. The CFD analysis under predicts the total resistance compared to model test. However various aspects of the appendage geometry were simplified for the CFD analysis and no free surface was included.

\section{CONCLUSIONS}

Three AUV's have been modelled in CFX to determine straight line resistance. This has then been validated against existing experimental data. The ease with which shape/appendage modifications where made as well as inclusion of the free surface demonstrates the success of the parametrisation strategy and its suitability for considering concept hull forms. CFD can be applied to the design of AUVs specifically:

- to determine straight line resistance of bare and appended hull forms;

- to allow rapid comparison of the resistance of different hull forms at the initial design stage through the use of highly parameterised geometric models;

- to complement model test experiments, to gain a clearer understanding of the origins of measured drag and to help understand the effect of test conditions on the open water performance of these vehicles;

- to provide detailed information about the mean flow pattern around the hull which would not normally be determined from standard towing tank experiments.

\section{ACKNOWLEDGEMENTS}

Dr Turnock acknowledges the Royal Academy of Engineering for a Global Research Award for his visit to Memorial University in the summer of 2004. Mr Phillips' PhD studentship is jointly financed by the School of Engineering Science and the National Oceanography Centre, Southampton.

\section{REFERENCES}

[1] G. Griffiths, Technology and Applications of Autonomous Underwater Vehicles. Taylor and Francis, 2002.

[2] S. F. Hoerner, Fluid-Dynamic Drag. Published by the Author, 1965.

[3] A. CFX, ANSYS CFX, Release 10.0. ANSYS Ltd, 2005.

[4] D. C. Wilcox, Turbulence Modeling for CFD. La Cnada, Calif. : DCW Industries, 1998.

[5] CFX, "Innovative turbulence modeling: Sst model in ansys cfx," ANSYS Ltd, Tech. Rep., 2006.

[6] ITTC, ITTC Quality Maual - CFD General Uncertainty Analysis in CFD Guildines for RANS codes. ITTC, 1999.

[7] P. Statham, D. Connelly, C. German, T. Brand, J. Overnell, E. Bulukin, N. Millard, S. McPhail, M. Pebody, J. Perrett, S. M, P. Stevenson, and A. Webb, "Spatially complex distribution of dissolved manganese in a fjord as revealed by high-resolution in situ sensing using the autonomous underwater vehicle autosub," Environmental Science and Technology, 2005.

[8] P. Wadhams, J. Wilkinson, and S. McPhail, "A new view of the underside of arctic sea ice," Geophysical Research Letters, 2006.

[9] N. Kimbler and W. Marshfield, "Design and testing of control surfaces for the autosub demonstrator test vehicle," DRA Haslar, Tech. Rep., 1993.

[10] C. Fallows, "Characterisation of the propulsion system of autonomous underwater vehicles," Ph.D. dissertation, University of Southampton, 2004.

[11] C. Pashias, Shape Optimisation of a Long Range Autonomous Underwater Vehicle Using Computational Fluid Dynamics, Ship Science Part III Honours Report SS583, 2001.

[12] R. Thomas, N. Bose, and C. D. Williams, "Propulsive performance of the autonomous underwater vehicle c-scout," in Oceans '2003 Conference Proceedings, 2003.

[13] S. Andersson, J. Morton, A. Gibbins, T. Palmer, E. Khoo, G. Stuebner, I. Lovatt, and S. O'Connell, European AUV 1 - Autonomous Underwater Vehicle for SAUC-E 2006 I \& II, GDP Report,. University of Southampton, 2006. 